\title{
SCALE FIXING BY DIMENSIONAL TRANSMUTATION: SUPERSYMMETRIC UNIFIED MODELS AND THE RENORMALIZATION GROUP
}

\author{
M.B. EINHORN and D.R.T. JONES \\ Randall Laboratory of Physics, University of Michigan, Ann Arbor, Michigan 48109, USA
}

Received 8 June 1982

(Revised version received 6 July 1982)

\begin{abstract}
We show how the renormalization group may be used in supersymmetric models to determine the expectation values of fields whose scales are undetermined by the classical potential. We apply this formalism to supersymmetric models of the O'Raifeartaigh type, and in particular. to Witten's model of the gauge hierarchy. We discuss the possibilities for a mass hierarchy and propose a criterion for an aesthetic model, which limits the magnitude of the hierarchies in perturbative unification models. We consider the zero-mass limit of Witten's model and argue that, despite the ground state becoming supersymmetric, the scale of the spontaneous symmetry breakdown of gauge symmetry remains fixed by radiative corrections. These models, after dimensional transmutation, undergo decoupling at smaller scales. In conclusion, we suggest the possibility of a new "weak tumbling" scenario for aesthetically generating mass and possibly gauge hierarchies. We also indicate how our work may be extended to finite temperatures and discuss the implications for cosmology.
\end{abstract}

\section{Introduction}

Globally supersymmetric unified models (SUMs) offer hope for resolving the naturalness or gauge hierarchy puzzles of grand unified theories. [1] A particularly attractive class of models was addressed by Witten [2], in which the fundamental lagrangian contains a single scale $m$ which represents the scale of spontaneous breakdown of supersymmetry by the mechanism originally suggested by O'Raifeartaigh [3]. However, in the tree approximation, there is a field (or fields) $X$ whose expectation value $\langle X\rangle$ remains undetermined, a necessary consequence of the O'Raifeartaigh mechanism. However, $\langle X\rangle$ may be determined by radiative corrections to the effective potential. In non-gauge theories, $\langle X\rangle$ remains small [4], but it was shown in ref. [2] that, in local gauge theories, this was not necessarily the case. In fact, it was plausibly argued that, if certain relations obtained between non-gauge couplings and gauge coupling constants, the scale of $\langle X\rangle$, determined by radiative corrections, could be exponentially larger than the original input scale $m$. If $\langle X\rangle$ also corresponds to the scale of gauge symmetry breaking, then the model naturally develops a hierarchy in which one symmetry breaking occurs at scale $\langle X\rangle$ and 
another occurs at scale $m \ll\langle X\rangle$. It was subsequently realized ${ }^{\star}$ that these models have many light particles (including scalars!) with masses on the order of $m^{2} /\langle X\rangle$. It was originally suggested [2] that $m$ be identified with the electroweak breaking of $\mathrm{SU}(2) \times \mathrm{U}(1)$ and $\langle X\rangle$ with the unification mass. An alternative possibility is that the electroweak scale is $m^{2} /\langle X\rangle$ while $m$ represents some intermediate scale of order $10^{9}-10^{11} \mathrm{GeV}$, assuming $\langle X\rangle\left\langle m_{\text {Planck }} \approx 10^{19} \mathrm{GeV}\right.$. The latter possibility has been termed the "geometric hierarchy" and recently developed into an apparently phenomenologically acceptable SUM [6].

It might appear at first sight that the unification scale is not reliably calculable in perturbation theory, because of the development of large logarithms $\ln X / m$. We shall show, however, that since the breakdown of perturbation theory is associated with a large change in scale rather than a large coupling constant, it can in fact be overcome by application of the renormalization group to the effective potential. The unification mass $\langle X\rangle$ is calculable (if it exists) so long as the coupling constants remain small on that scale, much as in standard GUTs.

To be specific, the prototypical model presented by Witten is an SU(5) gauge theory with three chiral supermultiplets: $A$ and $Y$ in the adjoint representation, and a singlet field $X$. The superpotential is

$$
W=\lambda_{1} \operatorname{Tr} A^{2} Y+\lambda_{2} X\left(\operatorname{Tr} A^{2}-m^{2}\right) .
$$

In tree approximation, one finds that

$$
\begin{aligned}
& \langle A\rangle=\frac{\lambda_{2} m}{\sqrt{\lambda_{1}^{2}+30 \lambda_{2}^{2}}} \operatorname{diag}(2,2,2,-3,-3), \\
& \langle Y\rangle=\frac{\lambda_{2}\langle X\rangle}{\lambda_{1}} \operatorname{diag}(2,2,2,-3,-3) .
\end{aligned}
$$

The scale of $\langle X\rangle$ is completely undetermined, but if $\langle Y\rangle\rangle\langle A\rangle$, then this sets the scale at which $\mathrm{SU}(5) \rightarrow \mathrm{SU}(3) \times \mathrm{SU}(2) \times \mathrm{U}(1)$. If one inputs the tree values, then the effective potential in one-loop approximation is given for $X \gg m$ by [2]

$$
V(X)=\frac{\lambda_{1}^{2} \lambda_{2}^{2} m^{4}}{\lambda_{1}^{2}+30 \lambda_{2}^{2}}\left[1+\frac{3 \hbar}{8 \pi^{2}}\left(\frac{\lambda_{2}^{2}}{\lambda_{1}^{2}+30 \lambda_{2}^{2}}\right)\left(29 \lambda_{1}^{2}-50 g^{2}\right) \ln \frac{X^{2}}{\mu^{2}}\right],
$$

where $g$ is the $\mathrm{SU}(5)$ gauge coupling constant and $\mu$ is a scale parameter introduced in the process of renormalization ${ }^{\star \star}$. If $50 \mathrm{~g}^{2}>29 \lambda_{1}^{2}$ on scale $\mu$, then one can only

* This apparently was originally realized by Banks (unpublished, see ref. [5]) and independently by Raby (private communication).

$\star \star$ We caution the reader that our notation differs from Witten [2]. 
conclude that the minimum in $X$, if it exists, is very much larger than $\mu$. If, on the other hand, one specifies the parameters of the theory on a large scale $\mu$ where $50 g^{2}<29 \lambda_{1}^{2}$, then the minimum, if it exists, will be smaller than $\mu$. Thus, one would expect a minimum to occur on a scale $m_{\mathrm{U}}$ where $50 g^{2}\left(m_{\mathrm{U}}\right)=29 \lambda_{\mathrm{I}}^{2}\left(m_{\mathrm{U}}\right)$, and $\langle X\rangle \approx m_{\mathrm{U}}$. The rigorous analysis below will justify this heuristic argument and show that normalizing at $m_{\mathrm{U}}$ one can calculate perturbatively.

The outline of the remainder of the paper is as follows. In sect. 2, we present a general formalism for applying the renormalization group to the effective potential. We also derive a general formula for $\operatorname{Tr}(-)^{\mathrm{F}} M^{4}$ for an arbitrary supersymmetric gauge theory. We obtain a simple rule for determining the minima which may exist at finite values of the fields and show how it may be applied through two loops $\left(O\left(\hbar^{2}\right)\right)$. We also present explicit formulas for the one-loop $\beta$-functions and anomalous dimensions for an arbitrary SUM. In sect. 3 we apply the formalism to Witten's model [2], derive the condition for a minimum to exist at finite $X$, and plot the characteristic trajectories for this model. We analyze five different physical theories, depending on the choice of parameters, and show under what circumstances a hierarchy of masses might arise. We also propose a technical definition of "aesthetic" (as distinct from "natural") which limits the acceptable magnitude of the hierarchy in a weak coupling theory. In sect. 4 , we discuss the limit of Witten's model when the mass $m \rightarrow 0$. The ground state becomes supersymmetric; nevertheless, the scale of gauge symmetry breaking remains fixed by radiative corrections. Regardless of the value of $m$, one must perform dimensional transmutation [7] in theories of this type in order to apply standard decoupling theorems. In sect. 5 we summarize our conclusions and make some speculations. We propose a new tumbling scenario [8] which does not require strong couplings. We also indicate how our work may be extended to finite temperatures and discuss some of its implications for the cosmology of these models ${ }^{\star}$.

\section{The effective potential and the renormalization group}

A general supersymmetric gauge theory will be described by a group $G$ and some chiral superfields $\phi_{i}$ transforming according to some (generally reducible) representation $R$ of $G$. The superpotential for a renormalizable theory will be a cubic polynomial

$$
W=a^{i} \phi_{i}+\frac{m^{i j}}{2} \phi_{i} \phi_{j}+\frac{\lambda^{i j k}}{3} \phi_{i} \phi_{j} \phi_{k}
$$

\footnotetext{
« While completing this work, we received a preprint by Yamagishi [9] treating the same topic and containing many of the same results presented here in sects. 2 and 3. However, our point of view about the hierarchy and decoupling (sect. 4) seems quite different from his. On the other hand, he has presented the explicit mass spectrum and obtained the low-energy effective superpotential which are not contained here. Finally, we accept full responsibility for the speculations of sect. 5 .
} 
The classical potential is given by

$$
V=\sum_{i}\left|\frac{\partial W}{\partial \phi_{i}}\right|^{2}+\frac{g^{2}}{2} \sum_{a} D_{a}^{2}
$$

For notational simplicity, we have assumed $\mathrm{G}$ is simple, but the formulation here can readily be extended to the general case. Thus, $g$ is the gauge coupling constant and $D_{a} \equiv \phi^{i}\left(T_{a}\right)_{i}^{j} \phi_{j}$, where $T_{a}$ represents the generators for $\mathrm{R}$, and $\phi^{i} \equiv \phi_{i}^{*}$. The one-loop approximation to the effective potential [7] may be taken to be

$$
V_{1}=V_{\mathrm{cl}}\left(\phi_{i}\right)+\frac{\hbar}{64 \pi^{2}} \operatorname{Tr}(-)^{\mathrm{F}} M^{4} \ln \frac{M^{2}}{\mu^{2}}
$$

where the second term is an abbreviated notation for the sum over scalars and vector bosons minus the sum over fermions. The mass matrices $M$ are determined in tree approximation as functions of the classical fields $\phi_{i}$. In stating that the second term is the complete and precise one-loop correction, we have tacitly assumed a specific mass-independent renormalization prescription ${ }^{\star}$. The exact effective potential $V_{\text {eff }}$, to all orders, obeys the renormalization group equation ${ }^{\star \star}$

$$
\left\{\mu \frac{\partial}{\partial \mu}+\beta_{\mathrm{g}} \frac{\partial}{\partial g}+\left[\beta^{i j k} \frac{\partial}{\partial \lambda^{i j k}}+\beta^{i j} \frac{\partial}{\partial m^{i j}}+\beta^{i} \frac{\partial}{\partial a^{i}}-\gamma_{i}^{j} \phi_{j} \frac{\partial}{\partial \phi_{i}}+\text { c.c. }\right]\right\} V_{\mathrm{eff}}=0
$$

We require a more compact notation for the parameters of the theory. Define

$$
\lambda_{\mathrm{p}} \equiv\left(g, \lambda^{i j k}, \lambda_{i j k}, m^{i j}, m_{i j}, a^{i}, a_{i}\right)
$$

so eq. (2.4) can be abbreviated as

$$
\left\{\mu \frac{\partial}{\partial \mu}+\beta_{\mathrm{p}} \frac{\partial}{\partial \lambda_{\mathrm{p}}}-\left[\gamma_{i}^{j} \phi_{j} \frac{\partial}{\partial \phi_{i}}+\text { c.c. }\right]\right\} V_{\mathrm{eff}}=0
$$

\footnotetext{
* We believe this is the simplest possible prescription, $c f$. ref. [7]. A single scale parameter $\mu$ has been introduced. In the non-supersymmetric case, one must generally subtract another term of order $h$ obtained from the one given in (2.3) by setting all coupling constants equal to zero in the expressions for $M^{2}$.

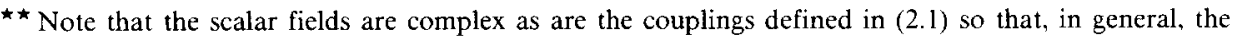
complex conjugate (c.c.) must be added. As is customary, we represent this by differentiating separately with respect to a quantity and its c.c. Care must be exercised to take into account that $m^{i j}$ and $\lambda^{i j k}$ are totally symmetric tensors. Group invariance implies the additional constraints $W^{i}\left(T_{a}\right)_{i}^{j} \phi_{j}$ $=0$ for all values of $\phi_{i}$, and derivatives of this equation are also useful. Throughout the paper, we assume that calculations are performed in the Landau gauge to avoid the appearance of a gauge parameter in (2.4) and elsewhere.
} 
In particular, it follows from (2.3) that, to one-loop order,

$$
\left\{\beta_{\mathrm{p}} \frac{\partial}{\partial \lambda_{\mathrm{p}}}-\left[\gamma_{i}^{j} \phi_{j} \frac{\partial}{\partial \phi_{i}}+\text { c.c. }\right]\right\} V_{\mathrm{cl}}=\frac{\hbar}{32 \pi^{2}} \operatorname{Tr}(-)^{\mathrm{F}} M^{4}
$$

We shall see below that the right-hand side will be of great importance in determining the extrema of the effective potential, so it will extremely useful to have a general formula for it. After considerable algebra, we find ${ }^{\star}$

$$
\begin{aligned}
\frac{1}{32 \pi^{2}} \operatorname{Tr}(-)^{\mathrm{F}} M^{4}= & \frac{1}{16 \pi^{2}}\left\{W^{i} W_{i j k} W^{j k l} W_{l}\right. \\
& +g^{2}\left[2 D_{a} W^{k i}\left(T_{a}\right)_{i}^{j} W_{j k}-2 \operatorname{Re} W_{k} W^{k i}\left(T^{2}\right)_{i}^{j} \phi_{j}-4 W^{i}\left(T^{2}\right)_{i}^{j} W_{j}\right] \\
& \left.+g^{4}\left[2 D_{a} \phi^{i}\left(T^{2} T_{a}\right)_{i}^{j} \phi_{j}+\left(T(R)-3 C_{2}(G)\right) \sum D_{a}^{2}\right]\right\}
\end{aligned}
$$

Here, we introduce an obvious notation for derivatives of the superpotential,

$$
W^{i} \equiv \frac{\partial W}{\partial \phi_{i}}, \quad W^{i j} \equiv \frac{\partial^{2} W}{\partial \phi_{i} \partial \phi_{j}}, \quad \text { etc. },
$$

and denote complex conjugation by raising or lowering indices, thus $\phi^{i} \equiv \phi_{i}^{*}$, $W_{i j}=W^{i j *}$, etc. The expression takes on a simpler appearance if we decompose $\mathrm{R}$ into its irreducible representations $\mathrm{T}_{a}=\Sigma_{\alpha} \oplus \mathrm{T}_{a}^{\alpha}$, so that $T^{2}=\Sigma_{\alpha} \oplus C_{2}\left(\mathrm{R}_{\alpha}\right) E_{\alpha}$, where $E_{\alpha}$ is the projector onto the irreducible representation $\mathrm{R}_{\alpha}$. Then we may write

$$
\begin{aligned}
\frac{1}{32 \pi^{2}} \operatorname{Tr}(-)^{\mathrm{F}} M^{4}= & \frac{1}{16 \pi^{2}}\left\{4 W^{i} \lambda_{i}^{(2) j} W_{j}+g^{2}\left[2 D_{a} W^{k i}\left(T_{a}\right)_{i}^{j} W_{j k}\right.\right. \\
& \left.-2 \sum_{\alpha} C_{2}\left(\mathrm{R}_{\alpha}\right) \operatorname{Re} W_{k} W^{k i}\left(E_{\alpha}\right)_{i}^{j} \phi_{j}-4 \sum_{\alpha} C_{2}\left(\mathrm{R}_{\alpha}\right) W^{i}\left(E_{\alpha}\right)_{i}^{j} W_{j}\right] \\
& \left.+g^{4}\left[2 \sum_{\alpha} C_{2}\left(\mathrm{R}_{\alpha}\right) D_{a} \phi^{i}\left(T_{a}^{\alpha}\right)_{i}^{j} \phi_{j}+\left(T(\mathrm{R})-3 C_{2}(\mathrm{G})\right) D_{a}^{2}\right]\right\}
\end{aligned}
$$

\footnotetext{
* We differ slightly from Yamagishi's formula [9]. Incidentally, this formula is true for an arbitrary superpotential and is not restricted to renormalizable theories. Finally, we remark that, since (2.6) is an identity in the fields, (2.7) provides a powerful check on the one-loop coefficient functions $\beta$ and $\gamma$. Some are related by non-renormalization theorems and so, given partial knowledge of these coefficients, the remainder can of ten be determined from (2.6) and (2.7)
} 
where we defined $\lambda_{i}^{(2) j} \equiv W_{i k l} W^{j k l} / 4$. It may be useful to recall that $D_{a} \equiv \Sigma_{\alpha} \phi^{i}\left(T_{a}^{\alpha}\right)_{i}^{j} \phi_{j}$ and $T(\mathrm{R})=\Sigma_{\alpha} d\left(\mathbf{R}_{\alpha}\right) C_{2}\left(\mathbf{R}_{\alpha}\right) / r$ where $d\left[\mathbf{R}_{\alpha}\right]$ is the dimension of $\mathbf{R}_{\alpha}$ and $r=d[\mathrm{G}]$ is the dimension of the group $\mathrm{G}$. Note that (2.8) vanishes as expected in the supersymmetric case where $D_{a}=0$ and $F_{i}^{*} \equiv-W^{i}=0$. Except in a gauge theory, $\operatorname{Tr}(-)^{\mathrm{F}} M^{4}$ $\geqslant 0$.

Now we shall return to the exact renormalization group equation (2.5) and discuss its general solution. Dimensional analysis implies

$$
\left[\mu \frac{\partial}{\partial \mu}+\delta_{\mathrm{p}} \lambda_{\mathrm{p}} \frac{\partial}{\partial \lambda_{\mathrm{p}}}+\phi_{i} \frac{\partial}{\partial \phi_{i}}+\phi^{i} \frac{\partial}{\partial \phi^{i}}-4\right] V_{\mathrm{eff}}=0
$$

where $\delta_{\mathrm{p}}$ is the dimension of coupling $\lambda_{\mathrm{p}}\left(\delta_{\mathrm{p}}=0\right.$ for $g, \lambda^{i j k} ; 1$ for $m^{i j} ; 2$ for $\left.a^{i}\right)$. Then, subtracting from (2.5), we obtain

$$
\left[-\left(\gamma_{i}^{j}+\delta_{i}^{j}\right) \phi_{j} \frac{\partial}{\partial \phi_{i}}+\text { c.c. }+\varphi_{1}\right] V_{\text {eff }}=0
$$

where

$$
\text { OD } \equiv\left(\beta_{\mathrm{p}}-\delta_{\mathrm{p}} \lambda_{\mathrm{p}}\right) \frac{\partial}{\partial \lambda_{\mathrm{p}}}+4
$$

For the typical problem of interest here, the minimum of certain fields are not determined at the classical level. For simplicity, let us suppose, as in Witten's model, there is a single such field $X$. Given $X$, all other fields are determined in the tree approximation. In the exact effective potential $V_{\text {eff }}$, we formally input the values of the exact minima (which may depend on $X$ ) for all fields other than $X$. Thus $\partial V_{\mathrm{eff}} / \partial \dot{\phi}_{i}=0$, so

$$
\left.\left\{-\left(1+\gamma_{X}\right) X \frac{\partial}{\partial X}+\text { c.c. }+\mathscr{D}\right)\right\} V=0
$$

where

$$
\gamma_{X} \equiv \gamma_{X}^{X}+\sum_{i \neq X} \frac{\left\langle\phi_{i}\right\rangle}{X} \gamma_{X}^{i}
$$

and $V$ is the value of $V_{\text {eff }}$ evaluated at the minima $\left\langle\phi_{i}\right\rangle$. We suppose further that $X$ may be taken to real, i.e., that one can choose phases such that $\gamma_{X}$ is real. The general solution of this first-order partial differential equation may be obtained by the method of characteristics [10]. 
Solve instead the system of ordinary differential equations

$$
-\mathrm{d} \tau=-\frac{\mathrm{d} X}{\left(1+\gamma_{X}\right) X}=\frac{\mathrm{d} \lambda_{\mathrm{p}}}{\beta_{\mathrm{p}}-\delta_{\mathrm{p}} \lambda_{\mathrm{p}}}=-\frac{\mathrm{d} V}{4 V},
$$

subject to initial conditions at $\tau=0$ which we take to be

$$
\lambda_{\mathrm{p}}=\Lambda_{\mathrm{p}}, \quad X=\hat{X}\left(\Lambda_{\mathrm{p}}\right), \quad V=\hat{V}\left(\Lambda_{\mathrm{p}}\right)
$$

The choice of $\hat{X}$ is a matter of convenience ${ }^{\star}$; common practice is to choose $\hat{X}=\mu$. The specification of $\hat{V}$ determines the dynamics of the theory but depends as well on the precise normalization conventions employed. Let us represent the solution for the parameters of the theory as

$$
\lambda_{\mathrm{p}}=\lambda_{\mathrm{p}}\left(\tau ; \Lambda_{\mathrm{p}^{\prime}}\right)
$$

Inverting this system yields the "running coupling constants"

$$
\Lambda_{\mathrm{p}}=\Lambda_{\mathrm{p}}\left(\tau ; \lambda_{\mathrm{p}^{\prime}}\right)
$$

They may equally well be defined as the solutions of

$$
\frac{\mathrm{d} \Lambda_{\mathrm{p}}}{\mathrm{d} \tau}=\left(\beta_{\mathrm{p}}\left(\Lambda_{\mathrm{p}}\right)-\delta_{\mathrm{p}} \Lambda_{\mathrm{p}}\right)
$$

subject to initial conditions that $\Lambda_{\mathrm{p}}=\lambda_{\mathrm{p}}$ at $\tau=0$. Then we must solve, subject to $X=\hat{X}\left(\Lambda_{\mathrm{p}}\right)$ at $\tau=0$,

$$
\frac{\mathrm{d} X}{\mathrm{~d} \tau}=\left(1+\gamma_{X}\right) X
$$

to obtain

$$
X=X\left(\tau ; \mu, \Lambda_{\mathrm{p}}\right)
$$

Inserting the running couplings from $(2.14 \mathrm{a})$ and then inverting determines

$$
\tau=\tau\left(X ; \mu, \lambda_{\mathrm{p}}\right)
$$

Finally the general solution for $V$ is (from (2.12))

$$
V=\hat{V}\left(\Lambda_{\mathrm{p}}\left(\tau ; \lambda_{\mathrm{p}^{\prime}}\right)\right) \exp (4 \tau)
$$

where we implicitly assume that the parameter $\tau$ is replaced by the function in $(2.16)$.

* The dependence of $\hat{X}$ and $\hat{V}$ on the scale parameter $\mu$ has been suppressed. 
It is tempting to try to write the inverse problem to (2.15), but since $\gamma_{X}$ may depend explicitly upon $X$, it is not so straightforward. It is instructive to work through the special case (sufficient for the application in the next section) when $\gamma_{X}$ depends only upon $\lambda_{p}$ and is independent of $X$. Then one can write

$$
\ln \frac{X}{\hat{X}\left(\Lambda_{\mathrm{p}^{\prime}}\right)}=\int_{0}^{\tau} \mathrm{d} \tau^{\prime}\left[1+\gamma_{X}\left(\lambda_{\mathrm{p}}\left(\tau^{\prime} ; \Lambda_{\mathrm{p}^{\prime}}\right)\right)\right]
$$

Replacing $\Lambda_{\mathrm{p}^{\prime}}$ by $\Lambda_{\mathrm{p}^{\prime}}\left(\tau ; \lambda_{\mathrm{p}}\right)$ and using the fact that

$$
\lambda_{\mathrm{p}}\left(\tau^{\prime} ; \Lambda_{\mathrm{p}^{\prime}}\left(\tau, \lambda_{\mathrm{p}}\right)\right)=\Lambda_{\mathrm{p}}\left(\tau-\tau^{\prime} ; \lambda_{\mathrm{p}^{\prime}}\right)
$$

we find

$$
\ln \frac{X}{\hat{X}\left(\Lambda_{\mathrm{p}}\left(\tau, \lambda_{\mathrm{p}^{\prime}}\right)\right)}=\int_{0}^{\tau} \mathrm{d} \tau^{\prime}\left[1+\gamma_{X}\left(\Lambda_{\mathrm{p}}\left(\tau^{\prime} ; \lambda_{\mathrm{p}^{\prime}}\right)\right)\right]
$$

In principle, $\Lambda_{\mathrm{p}}\left(\tau, \lambda_{\mathrm{p}^{\prime}}\right)$ is known, so the integral can be performed. This formula may be regarded as explicitly giving $X=X(\tau ; \hat{X} ; \lambda)$ or implicitly defining $\tau=$ $\tau\left(X ; \hat{X}, \lambda_{\mathrm{p}}\right)$. By analogy with the running coupling constants $(2.14 \mathrm{a})$, one might consider replacing $\hat{X}$ by a function $\bar{X}\left(\tau ; X, \lambda_{\mathrm{p}}\right)$. It obviously satisfies

$$
\frac{\mathrm{d} \bar{X}}{\mathrm{~d} \tau}=-\left(1+\gamma_{X}\right) \bar{X}
$$

Eq. (2.20a) generalizes to the case of an $X$-dependent anomalous dimension $\gamma_{X}$. The freedom previously expressed by the choice of the function $\hat{X}\left(\lambda_{\mathrm{p}}\right)$ now is expressed by the specification of an initial condition for $\bar{X}$. Although it may seem most natural to require $\bar{X}=X$ at $\tau=0$, one may equally well specify alternative initial conditions:

$$
\bar{X}=\hat{\bar{X}}\left(X, \lambda_{\mathrm{p}}\right) \quad \text { at } \tau=0
$$

Eqs. (2.20) are the inverse problem of (2.15) with appropriate choice for $\bar{X}$. (We find the original formulation less confusing.)

Now, we seek the minimum of $V$ for which

$$
J_{X} \equiv \frac{\partial V}{\partial X}=\frac{\partial \tau}{\partial X} \frac{\partial V}{\partial \tau}=\left(\frac{\partial \tau}{\partial X}\right) \mathrm{e}^{4 \tau}\left[\frac{\partial \hat{V}}{\partial \tau}+4 \hat{V}\right]=0
$$

Assuming the characteristic starting at $\tau=0$ actually reaches the minimum (which may require a judicious choice for $\hat{X}$ ) the prefactor $\partial \tau / \partial X \mathrm{e}^{4 r}$ will not vanish for 
finite $\tau^{\star}$, so the extrema satisfy

$$
J_{\tau}\left(\Lambda_{\mathrm{p}}\right) \equiv \frac{\partial \hat{V}}{\partial \tau}+4 \hat{V}=\left[\left(\beta_{\mathrm{p}}\left(\Lambda_{\mathrm{p}^{\prime}}\right)-\delta_{\mathrm{p}} \Lambda_{\mathrm{p}}\right) \frac{\partial}{\partial \Lambda_{\mathrm{p}}}+4\right] \hat{V}\left(\Lambda_{\mathrm{p}}\right)=0
$$

where we used the chain rule and $(2.14 \mathrm{~b})$. Alternatively, using dimensional analysis, the function $J_{\tau}\left(\lambda_{\mathrm{p}}\right)$ may be determined as

$$
J_{\tau}\left(\lambda_{\mathrm{p}}\right)=\mathscr{D} \hat{V}\left(\lambda_{\mathrm{p}}\right)=\left[\mu \frac{\partial}{\partial \mu}+\beta_{\mathrm{p}} \frac{\partial}{\partial \lambda_{\mathrm{p}}}\right] \hat{V}\left(\lambda_{\mathrm{p}}\right) .
$$

So the extrema are determined by calculating $J_{\tau}\left(\lambda_{\mathrm{p}}\right)$, replacing the parameters by their running couplings, and determining the zeros of $J_{\tau}\left(\Lambda_{\mathrm{p}}\left(\tau ; \lambda_{\mathrm{p}^{\prime}}\right)\right)$. The extrema so determined will be a local minimum provided that at those values of $X, \partial^{2} V / \partial X^{2}>0$. Generally,

$$
\frac{\partial^{2} V}{\partial X^{2}}=\left(\frac{\partial \tau}{\partial X}\right)^{2} \mathrm{e}^{4 \tau} \frac{\partial}{\partial \tau} J_{\tau}\left(\Lambda_{\mathrm{p}}\right)+\left(\frac{\partial \tau}{\partial X}\right) \mathrm{e}^{4 \tau} J_{\tau}\left(\Lambda_{\mathrm{p}}\right)\left(4 \frac{\partial \tau}{\partial X}+\frac{\partial^{2} \tau}{\partial X^{2}}\right)
$$

In the case of zeros at finite values of $\tau^{\star}$, where $J_{\tau}\left(\Lambda_{\mathrm{p}}\right)$ vanishes, only the first term contributes, and the requirement may be expressed more simply. Define

$$
H\left(\Lambda_{\mathrm{p}}\left(\tau ; \lambda_{\mathrm{p}^{\prime}}\right)\right) \equiv\left(4+\frac{\partial}{\partial \tau}\right) J_{\tau}\left(\Lambda_{\mathrm{p}}\right)
$$

Using the chain rule as before, we may write

$$
H\left(\lambda_{\mathrm{p}}\right)=\mathscr{Q}_{\tau}\left(\lambda_{\mathrm{p}}\right)=\left(\mu \frac{\partial}{\partial \mu}+\beta_{\mathrm{p}} \frac{\partial}{\partial \lambda_{\mathrm{p}}}\right) J_{\tau}\left(\lambda_{\mathrm{p}}\right)
$$

Thus the zeros of $J_{\tau}\left(\Lambda_{\mathrm{p}}\left(\tau ; \lambda_{\mathrm{p}^{\prime}}\right)\right)$ are local minima provided that, at those points,

$$
H\left(\Lambda_{\mathrm{p}}\left(\tau ; \lambda_{\mathrm{p}^{\prime}}\right)\right)>0
$$

The preceding formulation is completely general and exact if we regard $\beta_{\mathrm{p}}$ and $\gamma_{X}$ as known.

It remains to determine the function $\hat{V}$ for any given model. Suppose we consider the one-loop approximation to the effective potential (2.3). This will be a valid approximation provided the dimensionless coupling constants are sufficiently small

\footnotetext{
* For zeros at $\tau= \pm \infty(\langle X\rangle=0$ or $+\infty)$, one must analyze $\partial V / \partial X$ itself and rely on (2.24) below for stability rather than (2.26). These cases can also be interesting.
} 
on the scale $\mu$ and that the second term is not large compared to the classical potential. This requires that the masses $M$ not be too large, that is, the scale of the fields $\phi_{i}$ must not be too large compared to $\mu^{\star}$. Note that small masses present no problem for $V_{\text {eff }}$, that is, there are not large infrared logarithms. So we may admit fields whose scales are small compared to $\mu$. We shall return to these points in sect. 3 below.

In passing from $V_{\text {eff }}$ to $V,(2.11)$, we input the exact minima for those fields other than $X$. In one-loop approximation, the position of the minima will be of the form $\phi_{i}^{(1)}=\phi_{i}^{c l}+\hbar \Delta \phi_{i}$, where $\phi_{i}$ denote the tree values for these minima and $\Delta \phi_{i}$ the shift due to the one-loop correction. However the value of the effective potential depends on $\Delta \phi_{i}$ only in $\mathrm{O}\left(\hbar^{2}\right)$ since

$$
V_{1}\left(X, \phi_{i}^{(1)}\right)=V_{1}\left(X, \phi_{i}^{\mathrm{cl}}\right)+\left.\hbar \Delta \phi_{i} \frac{\partial V_{\mathrm{cl}}}{\partial \phi_{i}}\right|_{\phi_{i}^{\mathrm{cl}}}+\mathrm{O}\left(\hbar^{2}\right),
$$

but, by definition,

$$
\left.\frac{\partial V_{\mathrm{cl}}}{\partial \phi_{i}}\right|_{\phi_{i}^{\mathrm{cl}}}=0
$$

Thus we realize the remarkable simplification that, to one-loop accuracy, we may evaluate $V_{1}$ at the tree values $\phi_{i}^{\text {cl }}(X)$ ! These $\phi_{i}^{\text {cl }}(X)$ may depend on the field $X$ whose minimum is not determined at the classical level. Then $V_{1}$ has the form

$$
V_{1}=V_{\mathrm{cl}}\left(X, \phi_{i}(X), \ldots\right)+\frac{\hbar}{64 \pi^{2}} \operatorname{Tr}(-)^{\mathrm{F}} M^{4} \ln \frac{M^{2}}{\mu^{2}}
$$

In fact the value of the classical potential at the classical minima, the first term above, is completely independent of $X$ and is a function only of the parameters $\lambda_{\mathrm{p}}$, i.e., $V_{\mathrm{cl}}\left(X, \phi_{i}(X), \ldots\right) \equiv V_{0}\left(\lambda_{\mathrm{p}}\right)$. We conclude that the one-loop approximation to $V$ is

$$
V_{1}\left(X, \lambda_{\mathrm{p}}\right)=V_{0}\left(\lambda_{\mathrm{p}}\right)+\frac{h}{64 \pi^{2}} \operatorname{Tr}(-)^{\mathrm{F}} M^{4} \ln \frac{M^{2}}{\mu^{2}}+\mathrm{O}\left(\hbar^{2}\right)
$$

where the second term $\hbar \Delta V_{1}$ is to be evaluated by inserting the classical minima $\phi_{i}(X)$ for $i \neq X$. While the first term is independent of $X$, the second term will depend on $X$. To obtain $\hat{V}\left(\lambda_{\mathrm{p}}\right)$, we must choose $\hat{X}\left(\lambda_{\mathrm{p}}\right)$. Insert $X=\hat{X}\left(\lambda_{\mathrm{p}}\right)$ in (2.30),

* Although Witten [2] supposed this was true on the intrinsic scale $m$, of (1.3), it may or may not be the case that the fundamental couplings $g, \lambda^{i j k}$ are small on this scale. Actually, all that is required is to be able to find some scale $\mu$ on which all couplings are small. In practice, this may require $\mu \gg m$, since the fundamental coupling constant of SU(5) may increase substantially at small scales. 
that is,

$$
\hat{V}\left(\lambda_{\mathrm{p}}\right)=V_{1}\left(\hat{X}\left(\lambda_{\mathrm{p}}\right), \lambda_{\mathrm{p}}\right)=V_{0}\left(\lambda_{\mathrm{p}}\right)+\hbar \Delta V_{1}\left(\hat{X}\left(\lambda_{\mathrm{p}}\right), \lambda_{\mathrm{p}}\right)
$$

A particularly convenient choice for $\hat{X}$ is to make the second term zero, that is, choose $\hat{X}$ such that

$$
\operatorname{Tr}(-)^{\mathrm{F}} M^{4} \ln M^{2}=\left(\ln \mu^{2}\right) \operatorname{Tr}(-)^{\mathrm{F}} M^{4}
$$

In the large- $X$ limit, this corresponds to $\hat{X}=\mu$. Noting that $V_{0}$ is independent of $\mu$, we have

$$
J_{\tau}\left(\lambda_{\mathrm{p}}\right)=\beta_{\mathrm{p}} \frac{\partial V_{0}}{\partial \lambda_{\mathrm{p}}}\left(\lambda_{\mathrm{p}}\right)
$$

where the one-loop approximation to $\beta_{\mathrm{p}}$ is to be understood. There is a useful alternative expression for $J_{\tau}$. First, it is easy to see that

$$
\left.\frac{\partial V_{\mathrm{cl}}\left(X, \phi_{i}\right)}{\partial X}\right|_{\phi_{i}^{\mathrm{cl}}(X)}=0
$$

Now return to (2.6) and insert the tree values. Then we conclude that

$$
J_{r}\left(\lambda_{\mathrm{p}}\right)=\beta_{\mathrm{p}} \frac{\partial}{\partial \lambda_{\mathrm{p}}} V_{0}=\frac{\hbar}{32 \pi^{2}} \operatorname{Tr}(-)^{\mathrm{F}} M^{4}
$$

This implies, in particular, that $\operatorname{Tr}(-)^{\mathrm{F}} M^{4}$ is independent of $X$, even though $M^{2}$ depends on $X$ ! Now the right-hand side can be computed directly from our general expression (2.7) or (2.8). In certain cases, this too can be further simplified: From (2.2),

$$
V_{\mathrm{cl}}=W_{j} W^{j}+\frac{1}{2} g^{2} D_{a}^{2}
$$

So,

$$
\frac{\partial V_{\mathrm{cl}}}{\partial \phi_{i}}=W_{j} W^{i j}+g^{2} D_{a} \phi^{j}\left(T_{a}\right)_{j}^{i}
$$

If, as is true in Witten's model [2], the classical ground state has $D_{a}=0$, then it follows from (2.36) that $W_{j} W^{i j}=0$ also. In this case, (2.8) collapses to

$$
\frac{1}{32 \pi^{2}} \operatorname{Tr}(-)^{\mathrm{F}} M^{4}=\frac{1}{4 \pi^{2}}\left\{W^{i} \lambda_{i}^{(2) j} W_{j}-g^{2} \sum_{\alpha} C_{2}\left(\mathrm{R}_{\alpha}\right) W^{i}\left(E_{\alpha}\right)_{i}^{j} W_{j}\right\} .
$$


Recall also that, despite possible appearances to the contrary, it follows from (2.35) that (2.37) is independent of $X$.

Having determined the function $J_{\tau}\left(\lambda_{\mathrm{p}}\right)$, our rule is to replace $\lambda_{\mathrm{p}}$ by $\Lambda_{\mathrm{p}}\left(\tau ; \lambda_{\mathrm{p}^{\prime}}\right)$ and to find the solutions of $J_{\tau}\left(\Lambda_{\mathrm{p}}\left(\tau ; \lambda_{\mathrm{p}^{\prime}}\right)\right)=0^{\star}$. We remark that the existence of an extremum depends only upon determining that there exists a value of $\tau$ where $J_{\tau}$ vanishes, and not upon the precise relation between $\tau$ and $X$, and in particular not upon the choice for $\hat{X}$. If there is more than one minimum, one must select the one with the smallest value of the effective potential. In general, so long as the running coupling constants remain small, we expect the one-loop approximation to the effective potential to be good. From (2.31), it is given by

$$
V=V_{0}\left(\Lambda_{\mathrm{p}}\left(\tau ; \lambda_{\mathrm{p}^{\prime}}\right)\right) \mathrm{e}^{4 \tau}
$$

Of course, the actual degree of accuracy is unknown without performing a two-loop calculation. Presumably the existence of a zero of $J_{\tau}$ is independent of the choice of $\hat{X}$, even though the precise relationship between $\tau$ and $X$ will depend on this renormalization prescription. More generally, if we insert (2.31) into (2.23) and use (2.35), we find

$$
J_{\tau}\left(\lambda_{\mathrm{p}}\right)=\left.\hbar \mu \frac{\partial \hat{X}}{\partial \mu} \frac{\partial \Delta V_{1}}{\partial X}\right|_{X=\hat{X}}+\mathrm{O}\left(\hbar^{2}\right)
$$

The function $\hat{X}$ must be chosen so that $\partial \hat{X} / \partial \mu$ does not vanish; otherwise, the inversion from $\tau$ to $X$ implied in (2.16) cannot be carried out. Hence, to one loop, the zeros of $J_{\tau}$ are the solutions of

$$
\left.\frac{\partial \Delta V_{1}}{\partial X}\right|_{\substack{X=\hat{X} \\ \lambda_{\mathrm{p}}=\Lambda_{\mathrm{p}}}}=0
$$

We prefer to exploit the freedom of choice for $\hat{X}$ because (a) the zeros of (2.33) are explicitly a constraint among the parameters of the theory, and (b) eqs. (2.33) and (2.38) may be generalized to all orders. To see this, suppose the effective potential has been calculated through $N$ loops so that $(2.29)$ becomes

$$
V_{N}=V_{\mathrm{cl}}\left(X, \phi_{i}(X)\right)+\Delta V_{N}\left(X, \phi_{i}(X)\right)+\mathrm{O}\left(\hbar^{N+1}\right)
$$

Here $\phi_{i}(X)$ are the exact minima $\left(\phi_{i}(X)=\phi_{i}^{\mathrm{cl}}(X)+\Delta \phi_{i}(X)\right)$, but we may add and subtract $V_{\mathrm{cl}}\left(X, \phi_{i}^{\mathrm{cl}}(X)\right)$

$$
V_{N}=V_{\mathrm{cl}}\left(X, \phi_{i}^{\mathrm{cl}}(X)\right)+\Delta \tilde{V}_{N}\left(X, \phi_{i}(X)\right)+\mathrm{O}\left(\hbar^{N+1}\right),
$$

\footnotetext{
* This can occur in both abelian and non-abelian theories.
} 
that is, we absorb shifts from the classical minima in the radiative corrections. Suppose we choose $\hat{X}$ from the class of functions for which $(\partial / \partial \mu) \Delta \tilde{V}\left(\hat{X}, \phi_{i}(\hat{X})\right)=0$. With this choice equations (2.33) and (2.38) are formally correct to $\mathrm{O}\left(\hbar^{N+1}\right)$. Despite the complication this implies for determining the relation between $X$ and $\tau$, this choice allows the constraint among the parameters of the theory supplied by (2.33) to be determined to arbitrary accuracy from a knowledge of the $\beta$ alone!

It will clearly be necessary to determine the running couplings to solve any given model, so we need to determine the beta functions $\beta_{\mathrm{p}}$ and anomalous dimensions $\gamma_{j}^{i}$. To one-loop order, these are most easily determined in a mass-independent renormalization scheme, which has been assumed in (2.3) anyway. In one-loop, they are given by ${ }^{\star}$

$$
\begin{aligned}
& \beta_{\mathrm{g}}= \frac{g^{3}}{16 \pi^{2}}\left[-3 C_{2}(\mathrm{G})+\frac{1}{r} \sum_{\alpha} d\left[\mathrm{R}_{\alpha}\right] C_{2}\left(\mathrm{R}_{\alpha}\right)\right], \\
& \beta^{i j k}=\frac{1}{8 \pi^{2}}\left[\lambda_{l}^{(2) i} \lambda^{l j k}+\lambda_{l}^{(2) j} \lambda^{l k i}+\lambda_{l}^{(2) k} \lambda^{l i j}\right. \\
&\left.\quad-g^{2} \lambda^{i j k}\left(C_{2}\left(\mathrm{R}_{i}\right)+C_{2}\left(\mathrm{R}_{j}\right)+C_{2}\left(\mathrm{R}_{k}\right)\right)\right], \\
& \beta^{i j}= \frac{1}{8 \pi^{2}}\left[\lambda_{k}^{(2) i} m^{k j}+\lambda_{k}^{(2) j} m^{k i}-g^{2} m^{i j}\left(C_{2}\left(\mathrm{R}_{i}\right)+C_{2}\left(\mathrm{R}_{j}\right)\right)\right], \\
& \beta^{i}= \frac{1}{8 \pi^{2}}\left[\lambda_{j}^{(2) i} a^{j}-g^{2} a^{i} C_{2}\left(\mathrm{R}_{i}\right)\right], \\
& \gamma_{j}^{i}= \frac{1}{16 \pi^{2}}\left[2 \lambda_{j}^{(2) i}-g^{2} \delta_{j}^{i} C_{2}\left(\mathrm{R}_{i}\right)\right] .
\end{aligned}
$$

Notice that in a mass-independent renormalization scheme, the solution of the characteristic equations (2.12) naturally splits into subsets. The dimensionless running coupling constants form a closed system

$$
\frac{\mathrm{d} G}{\mathrm{~d} \tau}=\beta_{\mathrm{g}}(G, \Lambda), \quad \frac{\mathrm{d} \Lambda^{i j k}}{\mathrm{~d} \tau}=\beta^{i j k}(G, \Lambda)
$$

subject to initial conditions that $G=g$ and $\Lambda^{i j k}=\lambda^{i j k}$.

\section{Application to Witten's model}

In this section, we apply the machinery developed in the previous section to the SU(5) model discussed previously in sect. 1. In this model, we see from the first term

^ Refer to (2.8) for notation. 
in (1.3) that

$$
V_{0}=\frac{\lambda_{1}^{2} \lambda_{2}^{2} m^{2}}{\lambda_{1}^{2}+30 \lambda_{2}^{2}}
$$

Then the function $J_{\tau}\left(g, \lambda_{1}, \lambda_{2}, m\right)$ can be calculated from (2.37) (or simply read off from the second term in $(1.3)^{\star}$ )

$$
J_{\tau}=\left(\frac{3}{4 \pi^{2}}\right) \frac{\lambda_{1}^{2} \lambda_{2}^{4}\left(29 \lambda_{1}^{2}-50 g^{2}\right) m^{4}}{\left(\lambda_{1}^{2}+30 \lambda_{2}^{2}\right)^{2}} .
$$

Consequently, a zero of $J_{\tau}$ will occur where

$$
29 \Lambda_{1}\left(\tau ; \lambda_{\mathrm{p}}\right)^{2}=50 G\left(\tau ; \lambda_{\mathrm{p}}\right)^{2}
$$

It remains to be determined whether and where this occurs. For this purpose, we need to determine the $\beta$ functions and running couplings. For this model, one may readily obtain them by application of $(2.41)$ or by direct calculation. It is temporarily convenient to denote the gauge coupling by $\lambda_{0}=g$. Then the $\beta$ functions are of the form

$$
\beta_{\lambda_{p}}=\frac{\lambda_{\mathrm{p}}}{16 \pi^{2}} \sum_{q=0}^{2} b_{p q} \lambda_{q}^{2}, \quad p=0,1,2
$$

Generalizing slightly to $\operatorname{SU}(N)$, the matrix of coefficients is given by

$$
\left(\begin{array}{ccc}
-N & 0 & 0 \\
-6 N & \frac{5\left(N^{2}-4\right)}{N} & 8 \\
-4 N & \frac{4\left(N^{2}-4\right)}{N} & 2\left(N^{2}+3\right)
\end{array}\right)
$$

The anomalous dimension of the field $X$ is given by

$$
\gamma_{X}=\frac{1}{16 \pi^{2}} \gamma \lambda_{2}^{2}
$$

\footnotetext{
* As the derivation of (2.35) shows, this result for $J_{\tau}$ in no way depends on the scale of $X$ being large compared to $m$, despite the fact that the particular form given in (1.3) assumed this.
} 
with $\gamma=2\left(N^{2}-1\right)$. The anomalous dimension for the mass is then

$$
\beta_{m^{2}}=-\frac{m^{2}}{16 \pi^{2}}\left(b_{20} \lambda_{0}^{2}+b_{21} \lambda_{1}^{2}+\left(b_{22}-\gamma\right) \lambda_{2}^{2}\right)
$$

reflecting the fact that $\lambda_{2} m^{2} X$ is not renormalized. As described generally at the close of the preceding section, the solutions for $X(\tau ; \hat{X})$ and $M^{2}\left(\tau ; m^{2}\right)$ follow directly from the solutions for the dimensionless couplings, which themselves form a closed system of equations. In one-loop approximation, the equation for the gauge coupling $\lambda_{0}$ decouples from those for $\lambda_{1}$ and $\lambda_{2}$ and has the usual solution

$$
\frac{1}{G^{2}\left(\tau ; g^{2}\right)}=\frac{1}{g^{2}}-\frac{1}{8 \pi^{2}} b_{00} \tau
$$

The remaining two equations for the running couplings can be written as

$$
\begin{aligned}
& \frac{\mathrm{d} \Lambda_{1}^{2}}{\mathrm{~d} \tau}=\frac{\Lambda_{1}^{2}}{8 \pi^{2}}\left(b_{10} G^{2}+b_{11} \Lambda_{1}^{2}+b_{12} \Lambda_{2}^{2}\right), \\
& \frac{\mathrm{d} \Lambda_{2}^{2}}{\mathrm{~d} \tau}=\frac{\Lambda_{2}^{2}}{8 \pi^{2}}\left(b_{20} G^{2}+b_{21} \Lambda_{1}^{2}+b_{22} \Lambda_{2}^{2}\right) .
\end{aligned}
$$

The fact that $G^{2}(\tau ; g)$ is known and the homogeneity in $\Lambda_{i}^{2}$ suggests defining $Y_{i} \equiv \Lambda_{i}^{2} / G^{2}$. Then

$$
\frac{1}{G^{2}(\tau)} \frac{\mathrm{d} Y_{i}}{\mathrm{~d} \tau}=\frac{Y_{i}}{8 \pi^{2}}\left[b_{i j} Y_{j}-b_{00}\right], \quad i=1,2,
$$

where we define $Y_{0}=1$. This suggests a new choice for parameterizing the characteristic $\mathrm{d} \sigma \equiv\left(G^{2}(\tau) / 8 \pi^{2}\right) \mathrm{d} \tau$ which implies

$$
\sigma(\tau)=-\frac{1}{b_{00}} \ln \left(1-\frac{b_{00}}{8 \pi^{2}} g^{2} \tau\right)=\frac{1}{b_{00}} \ln \frac{G^{2}}{g^{2}},
$$

so that

$$
\frac{\mathrm{d} Y_{i}}{\mathrm{~d} \sigma}=Y_{i}\left[b_{i j} Y_{j}-b_{00}\right]
$$

with initial conditions $Y_{i}=\lambda_{i}^{2} / g^{2}$ at $\sigma=0$. Since the right-hand side of (3.12) now has no explicit dependence on $\sigma$, the characteristic trajectories in the $Y_{1}, Y_{2}$ plane are the solutions of

$$
\frac{\mathrm{d} Y_{2}}{\mathrm{~d} Y_{1}}=\frac{Y_{2}\left[b_{21} Y_{1}+b_{22} Y_{2}+\left(b_{20}-b_{00}\right)\right]}{Y_{1}\left[b_{11} Y_{1}+b_{12} Y_{2}+\left(b_{10}-b_{00}\right)\right]} .
$$




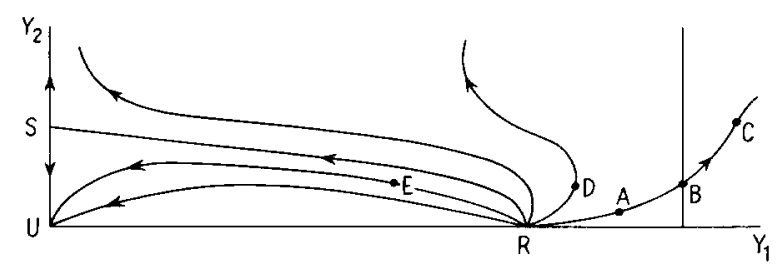

Fig. 1. Characteristic trajectories for Witten's model

TABLE 1

Liapounov exponents and eigenvectors for the fixed points in Witten's model

\begin{tabular}{ccc} 
& Exponent $\left(\mathrm{e}^{r \sigma}\right)$ & Eigenvector $\left(Y_{1}, Y_{2}\right)$ \\
\hline $\mathrm{U}:$ & $-3 N$ & $(0,1)$ \\
& $-5 N$ & $(1,0)$ \\
$\mathrm{S}:$ & $-\frac{N\left(5 N^{2}+3\right)}{N^{2}+3}$ & $\left(\begin{array}{c}3\left(N^{2}-4\right) \\
2 N\left(2 N^{2}+3\right)\end{array}\right)$ \\
& $+3 N$ & $(0,1)$ \\
$2 N$ & $\left(\begin{array}{c}2 N \\
N^{2}-4\end{array}\right)$ \\
& $+5 N$ & $(1,0)$ \\
\hline
\end{tabular}

The location of the fixed points is standard ${ }^{\star}$, being those values of $Y_{1}$ and $Y_{2}$ where both the numerator and denominator vanish in (3.13). These are

$$
\begin{array}{lll}
\mathrm{U}: & Y_{1}=0 & Y_{2}=0 \\
\mathrm{~S}: & Y_{1}=0 & Y_{2}=\left(b_{00}-b_{20}\right) / b_{22} \\
\mathrm{R}: & Y_{1}=\left(b_{00}-b_{10}\right) / b_{11} & Y_{2}=0 .
\end{array}
$$

Linearizing about each of these, we determine that $U$ is ultraviolet stable, $R$ is infrared stable, and $\mathrm{S}$ is a saddle point, so the characteristic trajectories are as depicted in fig. 1. The Liapounov exponents and eigenvectors are given in table 1. As one can see from table 1 , the unique trajectory which actually reaches the saddle point $\mathrm{S}$ has slope equal to

$$
\frac{\mathrm{d} Y_{2}}{\mathrm{~d} Y_{1}}=-\frac{3\left(N^{2}-4\right)}{2 N\left(2 N^{2}+3\right)}
$$

* These are called critical points in the mathematical literature; for a particularly nice discussion, see ch. 9 of ref. [11]. 
There is one unique characteristic which passes from $R$ to $S$ as indicated in fig. 1 . This determines the domain of attraction of the ultraviolet fixed point at the origin. We were unable to determine this trajectory analytically, but one can obtain it approximately by Liapounov's second method [11]. Presumably it could be easily determined if desired by numerical iteration starting at $\mathrm{S}$ with initial "velocity" $\mathrm{d} Y_{2} / \mathrm{d} Y_{1}$ equal to the critical slope given above.

We have been unable to make further progress toward an analytic solution for the running couplings. However, the running mass may be expressed in terms of them as ${ }^{\star}$

$$
\frac{\bar{m}^{2}}{m^{2}}=\mathrm{e}^{-2 \tau} \frac{\lambda_{2}}{\Lambda_{2}}\left[\left(\frac{\lambda_{1}}{\Lambda_{1}} \frac{G}{g}\right)^{4}\left(\frac{\Lambda_{2}}{\lambda_{2}}\right)^{5}\right]^{\left(N^{2}-1\right) /\left(5 N^{2}-1\right)} .
$$

Then $X$ is simply related; since the superpotential is not renormalized in a supersymmetric state, [12] $\lambda_{2} m^{2} X$ must scale canonically. Therefore

$$
\frac{X}{\hat{X}\left(\Lambda_{\mathrm{p}}\right)}=\mathrm{e}^{3 \tau} \frac{\Lambda_{2} \bar{m}^{2}}{\lambda_{2} m^{2}} .
$$

Obviously, the connection between $X$ and $\tau$ is generally quite complicated depending, among other things, on the choice for $\hat{X}\left(\Lambda_{\mathrm{p}}\right)$. Now the question of the existence of a solution of (3.3) corresponds, in the present language, to whether a characteristic curve intersects the line $Y_{1}=\frac{50}{29}$. This depends upon the specific initial values for $\lambda_{i}$ and $g$ as well as the intersection occurring for sufficiently small values of the running couplings so that the loop expansion is valid. To say this another way, the reliability of these calculations depends upon perturbative unification just as in standard GUTs. From fig. 1 , there will be some choices of $g, \lambda_{1}$, and $\lambda_{2}$ for which this will be true.

The theory is specified by giving $\mu, g, \lambda_{1}, \lambda_{2}$, and $m$. Although $\mu$ is arbitrary, we must assume that it can be specified in a range where the dimensionless couplings are all sufficiently small so that we can calculate perturbatively. Otherwise, we can say nothing at all. Now we may specify $m$ arbitrarily and, because the theory is supersymmetric, any value chosen will be technically natural. However, we would argue that it would be unaesthetic to choose a value for $m$ (except for the special case $m=0$ ) outside this range where all dimensionless couplings are small, because the physics on the scale $m$ is not describable perturbatively in terms of those coupling constants $^{\star \star}$. Given this definition of aesthetic, we may as well choose $\mu=m^{\star \star \star}$.

* We will denote the running mass in this model by $\bar{m}$ rather than by $M$, in order not to confuse it with the mass matrix $M$ of sect. 2 .

${ }^{\star}$ By this criterion, QED is aesthetic but QCD with current quark masses $m \ll \Lambda_{\mathrm{QCD}}$ is not aesthetic. This indicates that an unaesthetic model may not be complete but may yet be a phenomenologically useful theory.

$\star \star \star$ The reader who objects to our definition of unaesthetic can easily modify the following discussion by allowing $m$ to be an arbitrary scale independent of the normalization point $\mu$. Then additional cases are possible, but not qualitatively different. Indeed, in the next section, we shall consider a wider range for $m$. 
(The case $m=0$ is aesthetically acceptable only because it is a theory with a higher symmetry. In this respect, "aesthetic" agrees with "strongly natural". This case will be discussed in the next section.) Then we shall discuss five theories, depending on the values of the couplings on scale $m$ :

(A) Suppose $\lambda_{1}, \lambda_{2}$ and $g$ correspond to the point $\mathrm{A}$ in fig. 1. Since the minimum occurs at point $\mathrm{B}$, we have $\langle X\rangle>m$, so $\langle X\rangle$ determines the scale of $\mathrm{SU}(5)$ breaking (assuming $\lambda_{2}-\lambda_{1}$ ).

(B) Suppose $\lambda_{1}^{2}=\frac{50}{29} g^{2}$, and $\lambda_{2}$ is such that we are at point $\mathrm{B}$ in fig. 1 . Then $\langle X\rangle=m$ and there is a single mass scale.

(C) Suppose the initial values of the couplings place us at point $\mathrm{C}$ on scale $m$. Then $\langle X\rangle<m$, and so the gauge symmetry breaking is set by $m^{\star}$.

(D) Starting at point $\mathrm{D}$, the trajectory does not intersect the line at $Y_{\mathrm{l}}=\frac{50}{29}$ before the coupling $\lambda_{2}$ becomes large. In this case, we cannot trust perturbative calculations, so we cannot say what happens. But it seems likely that strong coupling will produce new physical phenomena on a scale $\gg m$.

(E) In this case, the initial values of these couplings are within the domain of attraction of the origin $U$. Then as $\tau \rightarrow \infty$, the theory is truly asymptotically free, with $\lambda_{1}$ and $\lambda_{2}$ tending to zero even more rapidly than $g$. Since no minimum is achieved for finite $\tau$, one cannot rely on $J_{\tau}\left(\lambda_{\mathrm{p}}\right)$ and $H\left(\lambda_{\mathrm{p}}\right)$ to tell us what is going on. However, there is no breakdown of perturbation theory so we can examine the behavior of the effective potential directly. Thus we want to examine as $\tau \rightarrow \infty$

$$
V=\mathrm{e}^{4 \tau} \bar{m}^{4} \frac{\Lambda_{1}^{2} \Lambda_{2}^{2}}{\Lambda_{1}^{2}+30 \Lambda_{2}^{2}} .
$$

Inserting our solutions for these quantities, this becomes

$$
V \sim \frac{m^{4}\left[Y_{2}^{30} Y_{1}^{7} G^{60}\right]^{1 / 31}}{Y_{1}+30 Y_{2}}
$$

From table 1, we find that, as $\tau \rightarrow \infty$,

$$
V \sim \tau^{-2} \rightarrow 0
$$

where we used (3.8) for $G$. Thus the effective potential decreases monotonically to zero as $\tau \rightarrow \infty$, or in terms of $X$, we find

$$
V-m^{4}\left(\ln \frac{X}{\hat{X}}\right)^{-2} \rightarrow 0 .
$$

\footnotetext{
* This possibility seems to have been ignored previously.
} 
We interpret this to mean that, as a result of radiative corrections, $\langle X\rangle=\infty$. We shall discuss this case further in the next section, where we shall argue that this is a perfectly acceptable vacuum state ${ }^{\star}$.

In addition to the above cases, one must also analyze the theory in the extreme ultraviolet $(X \rightarrow \infty)$ and infrared $(X \rightarrow 0)$. Except for case (E) above, either $\Lambda_{2}$ or $\Lambda_{1} \rightarrow \infty$ as $X \rightarrow \infty$, so perturbation theory breaks down. In the infrared, although $Y_{2} \rightarrow 0$ and $Y_{1} \rightarrow$ constant, $G$ blows up, so again perturbation theory breaks down. Thus, the preceding cases (A), (B), (C), and (E) exhaust the range of applicability of a perturbative analysis, even after summing up large logarithms by utilizing the renormalization group.

Incidentally, although it is intuitively clear, one can easily check that the $\langle X\rangle$ is, in fact, a local minimum in cases (A)-(C) by verifying that $H>0,(2.26)$.

Because the dimensionless couplings vary only logarithmically with scale, it is quite possible to generate a calculably large hierarchy, that is, it can turn out that $m \ll\langle X\rangle$ in case (A) and $m \gg\langle X\rangle$ in case (C). The case (E) is an example of the ultimate hierarchy, since $m /\langle X\rangle=0$. However, our analysis of the $m=0$ case in the next section suggests that, in some sense, the hierarchy has been put in by hand.

\section{Dimensional transmutation and decoupling}

In this section, we wish to argue that the scale of symmetry breaking $\langle Y\rangle$ and the scale $\langle X\rangle$ are not set by $m$. To see this, we shall first consider the $m \rightarrow 0$ limit of the theories defined by $W$. To be precise, imagine, in contrast to the discussion of the previous cases in sect. 3, we have normalized in such a way as to yield eq. (1.3) for $V_{\text {eff }}$ at an arbitrary scale $\mu$ and have specified $g, \lambda_{1}, \lambda_{2}$ and $m$. What happens as $m \rightarrow 0$ for fixed $g, \lambda_{1}, \lambda_{2}$, and $\mu$ ? Clearly, we have $\langle A\rangle \rightarrow 0$ and the ground state becomes supersymmetric. However, $\langle X\rangle$ remains fixed by the zero of $J_{\tau}$ which, in one-loop, corresponds to the relation

$$
29 \lambda_{1}^{2}(\langle X\rangle)=50 g^{2}(\langle X\rangle)
$$

Similarly, $\langle Y\rangle$ remains fixed and, in lowest order, in given by (1.2b). Thus, in the limit $m \rightarrow 0$, the gauge symmetry $\mathrm{SU}(5)$ breaks to $\mathrm{SU}(3) \times \mathrm{SU}(2) \times \mathrm{U}(1)$ at scale $\langle Y\rangle$. However, in this limit, the theory is specified by three dimensionless couplings $g, \lambda_{1}, \lambda_{2}$, and an arbitrary scale parameter $\mu$. Since the physical theory is independent of the choice of $\mu$, one should think of it as being specified by a one-dimensional parameter, $\langle X\rangle$ or $\langle Y\rangle$, determined by radiative corrections, and two dimensionless couplings, say $g$ and $\lambda_{2}$, with $\lambda_{1}$ fixed in terms of these. Although the scale of $V$ and $J_{\tau}$ are set by $m$, the position of the zeros of $J_{\tau}$ is independent of $m$ ! Certainly as $m \rightarrow 0$ and, we would argue, also for finite $m$, these theories are best

${ }^{\star} C f$. Witten [2] and Yamagishi [9]. 
thought of as undergoing dimensional transmutation, just as in the original illustration of the phenomenon [7].

Consider for a moment the theory de _ned by setting $m=0$ in $W$ (1.1) with superpotential

$$
W_{0}=\lambda_{1} \operatorname{Tr} A^{2} Y+\lambda_{2} X \operatorname{Tr} A^{2}
$$

This theory has a large class of degenerate supersymmetric ground states, including $A=0, X$ arbitrary, and $Y$ an arbitrary diagonal matrix. Thus one has symmetry breaking patterns other than $\mathrm{SU}(5) \rightarrow \mathrm{SU}(3) \times \mathrm{SU}(2) \times \mathrm{U}(1)$, and the scales of $\langle X\rangle$ and $\langle Y\rangle$ are arbitrary and unrelated. The non-renormalization theorems [12] assure us that any one of these remains correct to all orders in perturbation theory, that is, the superpotential is not renormalized. Nevertheless, it is apparent from the discussion above there is a unique one of these degenerate ground states which is selected by the $m \rightarrow 0$ limit of $W,(1.1)$. Thus, the addition of a term linear in $X$ to $W_{0}$, with arbitrarily small coefficient, determines the direction of the symmetry breaking to be $\mathrm{SU}(3) \times \mathrm{SU}(2) \times \mathrm{U}(1)$ and fixes the scales of $\langle X\rangle$ and, hence, $\langle Y\rangle$ by radiative corrections through dimensional transmutation. Since $m /\langle X\rangle$ can be arbitrarily small, this is the ultimate hierarchy.

Note by the way that the theory defined by $W_{0}$ is strongly natural and has a higher global symmetry than $W$, namely $A \rightarrow \mathrm{e}^{i \alpha} A$ with $X, Y$ unchanged. The original Witten model, $(1.1)$, is a theory in which this symmetry has been softly but explicitly broken down to $A \rightarrow-A$. The scale of this explicit breaking is $m$, is arbitrary, and bears no relation to the unification scale $\langle Y\rangle$, from this point of view. If $m \ll\langle X\rangle$, then the theory possesses a large hierarchy but it has been put in by hand, just as in standard GUTs. However, because this theory is supersymmetric on scales greater than $m$, there is no "naturalness" or "fine tuning" problem, that is, radiative corrections will not lift the scale up to $\langle X\rangle$. In this sense, Witten's model is natural but does not "explain" the hierarchy. On the other hand, even if $m$ is chosen aesthetically and the dimensionless couplings specified on that scale, there can be an enormous range for the resulting ratio $m /\langle X\rangle$, because the dimensionless couplings vary only logarithmically with scale. Thus if one chooses a theory "at random" by specifying "initial" values for the couplings on scale $m$, then it is clear from fig. 1 that one is apt to get $\langle X\rangle$ very different from $m$. However, it seems to us equally likely that $m \gg\langle X\rangle$ as $m \ll\langle X\rangle$.

Supposing that $m \ll\langle X\rangle$, let us discuss the effective theory on scales $\mu \ll\langle X\rangle$ but $\mu \geqslant m$. After dimensional transmutation, the usual decoupling arguments [13] apply. On these scales, the effective theory is supersymmetric and so will be describable by a $\mathrm{SU}(3) \times \mathrm{SU}(2) \times \mathrm{U}(1)$ symmetric gauge theory described by a superpotential $W_{\text {eff }}$ which will be a polynomial in the light fields containing terms which, beyond third order in the fields, have couplings proportional to inverse 
powers of $\langle X\rangle^{\star}$. There will be some supermultiplets of mass on the order of $m$ and others which are massless to order $m^{2} /\langle X\rangle^{\star \star}$, which sets the scale of the mass splittings among members of supermultiplets. In this sense, $m^{2} /\langle X\rangle$ sets the scale of supersymmetry breaking. To be more precise, the effective low energy theory on scales $m \ll \mu \ll\langle X\rangle$ will contain certain parameters $\kappa_{\mathrm{p}}$. These may be more or fewer than the number of parameters of the underlying theory. For example, there will be three gauge coupling constants $g_{3}, g_{2}, g_{1}$ corresponding to each unbroken gauge group $\mathrm{SU}(3) \times \mathrm{SU}(2) \times \mathrm{U}(1)$. The relation between these parameters $\kappa_{\mathrm{p}}$ and the parameters $\lambda_{\mathrm{p}}$ of the fundamental theory will be obtained in the standard manner by running the effective theory $u p$ to scale $\langle X\rangle$ via the beta functions of the effective theory and matching them to the parameters of the underlying theory. One does not run the parameters $\lambda_{p}$ down via the beta functions of the underlying theory to the scale of interest; in general, one can not even do this reliably because certain coupling constants (such as the $\mathrm{SU}(5)$ gauge coupling $g$ ) may become large, invalidating a perturbative calculation. Of course, this is irrelevant to the physics on scale $\mu$. To state this another way, the only scales $\mu$ on which the fundamental Lagrangian provides a good representation of the physical degrees of freedom is $\mu \geq\langle X\rangle$. It seems to us unattractive to introduce ad hoc a mass parameter $m$ into this theory with $m \ll\langle X\rangle$.

However unattractive it may seem, this is, we admit, a matter of taste and others may regard this an explanation for mass hierarchies. However, there is a limit to the magnitude of the hierarchy, for it is certainly unaesthetic, in the technical sense introduced in the previous section, to introduce a mass parameter on a scale on which any of the dimensionless couplings become of order unity, since there is no way to calculate reliably on that scale. However, this still allows considerably more freedom of choice in a fundamental theory than appeals to us. Perhaps the specification of the absolute magnitude of $m$ requires going beyond the SUM framework, to the inclusion of gravity, for instance. In that case, there is no aesthetic objection to any particular choice for $m$ and, because of supersymmetry, every choice is technically natural. We shall speculate about other possibilities in the next section.

In concluding this section, we would like to reiterate that it is only after dimensional transmutation that the standard decoupling theorems apply. If we renormalize on scale $\mu$, regarding $W$ as a theory with 3 dimensionless couplings $g$, $\lambda_{1}$, and $\lambda_{2}$, and one mass parameter, $m$, then $\langle X\rangle$ can be varied only by varying the dimensionless couplings in a certain way. That is not a situation in which decoupling arguments apply. Thus, it is imperative that we regard Witten's original model as a theory with two mass scales, $m$ and $\langle X\rangle$, and only two coupling constants, $g$ and $\lambda_{2}$.

^ Yamagishi [9] has given the leading (renormalizable) contributions to $W_{\text {eff }}$ and has worked out the fields and spectrum of the light theory.

${ }^{\star \star}$ See the first footnote on page 30 and appendix $A$ of ref. [6] for a discussion of the stronger decoupling theorem. 
Let us return to case $(\mathrm{E})$ of the preceding section, for which we found $\langle X\rangle=\infty$. We now realize that this is a theory in which decoupling is complete, and the low energy effective theory becomes the exact theory on all scales. The effect of radiative corrections has been to push some degrees of freedom in the classical theory off to infinity. Nevertheless, the vacuum is perfectly well-defined and stable, shifted in energy by only a finite amount from the classical energy. Thus it seems one can write down a Lagrangian apparently containing degrees of freedom at the classical level which are not present in the quantum theory ${ }^{\star}$.

\section{Summary and further speculations}

In this paper, we have generalized the application, initiated in ref. [7], of the renormalization group to the effective potential. This may have many applications to both condensed matter and elementary particle physics, but our particular motivation was the analysis of supersymmetric models of O'Raifeartaigh type. In sect. 2, we derived the general conditions for the determination of local minima, (2.22) and (2.26). We showed how the effective potential may be determined at all scales by matching to perturbation theory. In the class of models of interest, the minima of the effective potential for the field $X$ may be determined in principle through $N$ th order from the $N$-loop $\beta$ functions, knowing only the tree approximation to the positions of the other fields.

In sect. 3, we applied the formalism to Witten's SU(5) model [2] and discussed the characteristic curves for various assumptions about the parameters of the theory.

There is a certain paradox about the results which illustrates the power of the renormalization group. Suppose one had happened to choose to specify the theory on a scale $\mu$ for which $\lambda_{1}^{2}=50 g^{2} / 29$, and suppose further that $m \ll \mu$. Then we would have found that the effective potential remains independent of $X$ (for $X \sim \mu$ ) through one-loop order in perturbation theory. Since $V_{\text {eff }}$ is independent of $X$, one concludes that, if $X$ has a minimum on this scale, its determination requires going at least to two-loop order. How then were we able to conclude that $\langle X\rangle=\mu$ ? The resolution is that the curvature of the effective potential can only be seen in order $\hbar^{2}$. Indeed, one sees from eq. (2.5) that, whereas the leading non-zero approximation to $J_{\tau}$ is $\mathrm{O}(\hbar)$, the lowest non-trivial approximation to $H$ is $\mathrm{O}\left(\hbar^{2}\right)$, beyond the reach of a one-loop approximation to $V$. Had we had the foresight to normalize at this value of $\mu$, then by doing a second order calculation to $V_{\text {eff }}$ we should find

$$
\frac{\partial^{2} V}{\partial X^{2}} \propto H(X-\mu)^{2},
$$

so we could have concluded that $\langle X\rangle=\mu$ and avoided any discussion of the renormalization group. Thus the result can be realized perturbatively! Had we

\footnotetext{
* Of course, new physics such as gravity may cause one to modify the theory.
} 
normalized on any other scale $\mu \neq\langle X\rangle$, then we could only have concluded from perturbation theory that $\langle X\rangle \gg \mu$ or $\langle X\rangle \ll \mu$. The triumph of the renormalization group analysis is its ability to compensate for changes of scale and to allow a perturbative calculation starting from any scale as long as the running couplings begin small and remain small throughout the range of interest.

In sect. 4 , we considered the relationship between $m$ and $\langle X\rangle$ and took up decoupling. Considering the $m \rightarrow 0$ limit, we argued that $\langle X\rangle$ and $\langle Y\rangle$ remain fixed and finite, being set by a relation among dimensionless coupling constants. Thus, Witten's model is best regarded as having two scales, $m$ and $\langle X\rangle$, the second entering via dimensional transmutation [7].

Let us contrast the situation here with the more familiar Coleman-Weinberg $(\mathrm{CW})$ mechanism [7]. In the CW case, the scalar field is determined already at the tree level but, after radiative corrections, is shifted in such a way that the symmetry is reduced. In the O'Raifeartaigh case considered here, the scalar field or fields of interest remain undetermined at the classical level. Generally, the symmetry is already spontaneously broken, albeit at an arbitrary scale. It is this scale which is determined by radiative corrections in theories like Witten's model, (1.1). As in the original application [7], these theories are best regarded as undergoing "dimensional transmutation". (Note that at the minimum, the couplings $\lambda_{1}$ and $g$ are of the same order, rather than $\lambda_{1} \sim g^{2}$ as in the case of scalar electrodynamics.) We have argued that the discussion of decoupling, even in models which like Witten's [2] have an explicit scale, requires that one perform this exchange of a dimensional parameter for a dimensionless one. All this could conceivably occur in non-supersymmetric theories as well and is similar to the discussion of the masses of pseudo-Goldstone bosons [14].

Since $W(1.1)$ undergoes dimensional transmutation, the model is best regarded as a theory with two mass parameters, $\langle X\rangle$ and $m$, and with one fewer dimensionless coupling constant. (This is necessary in order to discuss decoupling.) In this sense, the theory is similar to the standard GUTs. It may be that $m \ll\langle X\rangle$ or $m \gg\langle X\rangle$, but such a hierarchy has in a sense been put in by hand just as in non-supersymmetric models or as in previous SUMs [1]. However, the logarithmic variation of the coupling constants with scale makes a large difference between $m$ and $\langle X\rangle$ more natural, as long as $m$ is chosen aesthetically.

We have tacitly assumed throughout this paper that $\lambda_{2}$ and $\lambda_{1}$ are of the same order on the scale of $\langle X\rangle$. If they were very different, then another mass hierarchy would develop since $\langle X\rangle$ and $\langle Y\rangle$ would be very different. As one sees from the figure, their behavior in the infrared (near $R$ ) is quite different since $Y_{2} / Y_{1} \rightarrow 0$ as $\tau \rightarrow-\infty$. Presumably this is irrelevant since this behavior has nothing to do with the physics on that scale. However, if one adopts the point of view of Witten [2], then another large hierarchy might seem natural, since, if $\lambda_{1} \sim \lambda_{2}$ initially at $m \ll\langle X\rangle$, then their evolution will be similar to the case D discussed in sect. 3 and indicated on the figure. Indeed, it seems one must initially specify $\lambda_{2} \ll \lambda_{1}$ in order to have 
perturbative unification. So there is a possibility of a substantial difference between $\langle X\rangle$ and $\langle Y\rangle$. It would be of interest to pursue this further, however, henceforth we shall assume $\lambda_{2} \sim \lambda_{1}$ at $\langle X\rangle$ and speak loosely of the scale of unification as either $\langle X\rangle$ or $\langle Y\rangle$.

Having said this, it is easy to imagine other scenarios which do lead to intrinsically large hierarchies. Suppose we begin with a supersymmetric theory like $W$ which, for simplicity, has only one mass parameter $m$ and which undergoes spontaneous breakdown of a gauge group $\mathrm{G}$ to another symmetry $\mathrm{G}_{1}$ at a scale $\langle X\rangle$ determined by a relation among dimensionless couplings. After dimensional transmutation, we may apply standard decoupling theorems to replace the fundamental Lagrangian $\mathcal{E}[\mathrm{G}]$ by an effective Lagrangian

$$
\varrho[G] \rightarrow \varrho\left[G_{1}\right]+\frac{1}{\langle X\rangle} \varrho_{\mathrm{NR}}+O\left(\frac{1}{\langle X\rangle^{2}}\right) .
$$

The first approximation to the low energy effective theory is a renormalizable Lagrangian $\mathcal{E}\left[\mathrm{G}_{1}\right]$ which, since supersymmetry remains unbroken, will be describable by some $\mathrm{G}_{1}$-invariant superpotential $W\left[\mathrm{G}_{1}\right]$. The form of $W\left[\mathrm{G}_{1}\right]$ will be much like $W[\mathrm{G}]$, and it may be that the process will repeat itself, that is, could undergo symmetry breaking at some other scale $\left\langle X_{1}\right\rangle$ determined by radiative corrections through the intersection of certain combinations of running coupling constants of $\mathcal{L}\left[\mathrm{G}_{1}\right]$. The scale $\left\langle X_{1}\right\rangle$ will be determined by the expectation value of some field $X_{1}$ in $W\left[G_{1}\right]$ which was undetermined classically. Because the couplings in $\mathcal{E}\left[G_{1}\right]$ vary only logarithmically, one could naturally anticipate large mass hierarchies ${ }^{\star}$. Moreover, this may lead to further gauge symmetry breaking $G_{1} \rightarrow G_{2}$. And the process may continue, in much the same manner as envisaged in strong-coupling tumbling scenarios [8]. The advantage here is that the entire process may occur for weak couplings and, therefore, may be calculable. Weak tumbling models will be explored in future work.

At present, it seems to be necessary to introduce an explicit scale parameter $m$ to initiate the weak tumbling and to determine its direction. It would have been nice not to have to introduce any mass parameter at all. From our point of view, the function of $m$ is to insert the scale of supersymmetry breaking into the theory. If supersymmetry breaking could be dynamically generated, it would serve the same function, but so far no one has provided a convincing illustration of its occurrence in four dimensions $[2,16]$. Another possibility is that of soft explicit breaking resulting from a low energy effective description of supergravity theories [17]. One might well hope that all masses would find their ultimate origin in at most one input scale.

In sect. 4 , we pointed out that the $m \rightarrow 0$ limit of Witten's model selects uniquely one of the continuum of degenerate ground states of $W_{0}$. This may be interpreted to

* This is similar to, but not the same as, a scenario previously proposed by Weinberg [15]. 
mean that the theory defined by $W_{0},(4.2)$, is extraordinarily sensitive to small changes in the theory. The addition of a term linear in $X$ is only one example. If one imagines $W_{0}$ is itself the effective field theory for an even more fundamental theory, it may be that additional higher dimensional operators select among the degenerate ground states, a situation in which "irrelevant operators" would not be irrelevant. If one generalizes Witten's model from $\mathrm{SU}(5)$ to $\mathrm{SU}(N)$, then for $N$ even, there are supersymmetric ground states with arbitrary values of $\langle X\rangle$. By the non-renormalization theorems [12], this remains true to all orders, so one is led to inquire about the effects of small changes in the models for even $N$. Since supersymmetry is broken at any finite temperature, there will be temperature-induced radiative corrections which might resolve the degeneracy.

The cosmology of these models is quite interesting and paradoxical. Temperature may be included in the renormalization group analysis by simply treating $T$ as another parameter with dimensions of mass but with anomalous dimension zero. Previous discussions of Witten's model argued that no symmetry breaking occurs until the temperature falls to $T \sim m$, at which point $\langle X\rangle$ may grow to a large value [18]. If, when $T$ falls to $O(m),\langle Y\rangle$ grows slowly compared to the expansion time, then one might expect that an intermediate era of exponential expansion would be initiated, as in one popular cosmology [19], which has recently been amended [20]. It remains to be worked out whether a marriage of these two ideas can really be arranged.

We would like to thank Y.-P. Yao for discussions about decoupling, and G.L. Kane and Y.-P. Yao for their comments on the manuscript. This research has been supported in part by the U.S. Department of Energy.

Note added in proof. In a recent paper [21], it is pointed out that in the model of Witten, which we used in sect. 3 to illustrate the formalism, starting values of the couplings very close to the infra-red fixed point $R$ are required to generate a hierarchy characteristic of grand unified theories, so a fine tuning is required after all. It can be seen, however, that this result is due to the accidental proximity of $\mathrm{R}$ to the line $Y_{1}=\frac{50}{29}$ and therefore not necessarily true of such models in general. We will present elsewhere an example which avoids this criticism.

A formula for $\operatorname{Tr}(-1)^{\mathrm{F}} M^{4}$ equivalent to (2.7) for the special case when $W$ is a cubic polynomial has been independently calculated by Barbieri et al. [22]. An analogous formula has also been given by Frampton et al. [23] but appears to differ from our (and Barbieri et al.'s) result by the omission of the second term in (2.8).

\section{References}

[1] S. Dimopoulos and S. Raby, Nucl. Phys. B192 (1981) 353;

M. Dine, W. Fischler and M. Srednicki, Nucl. Phys. B189 (1981) 575 ;

E. Witten, Nucl. Phys. B188 (1981) 513; 
S. Weinberg, Phys. Rev. D26 (1982) 287;

M. Dine and W. Fischler, Phys. Lett. 110 B (1982) 227; Nucl. Phys. B204 (1982) 346

S. Dimopoulos and H. Georgi, Nucl. Phys. B193 (1981) 150;

N. Sakai, Z. Phys. C11 (1982) 153

[2] E. Witten, Phys. Lett. 105B (1981) 267

[3] L. O'Raifeartaigh, Nucl. Phys. B96 (1975) 331

[4] M. Huq, Phys. Rev. D14 (1976) 3548

[5] E. Witten, Introduction to supersymmetry, Section V, Princeton preprint

[6] S. Dimopoulos and S. Raby, Los Alamos LA-UR-82-1282, May, 1982

[7] S. Coleman and E. Weinberg, Phys. Rev. D7 (1973) 1888

[8] S. Dimopoulos, S. Raby and L. Susskind, Nucl. Phys. B169 (1980) 373

[9] H. Yamagishi, Renormalization-group analysis of supersymmetric mass hierarchies, Princeton University preprint

[10] P.R. Garabedian, Partial differential equations (Wiley, N.Y., 1964)

[11] W.E. Boyce and R.C. DiPrima, Elementary differential equations and boundary value problems (2nd edition) (Wiley, N.Y., 1969)

[12] M.T. Grisaru, Four lectures on supergraphs, ICTP Spring School on Supergravity, Trieste, April 1981, and references therein;

E. Witten, Nucl. Phys. B185 (1981) 513

[13] K. Symanzik, Comm. Math. Phys. 34 (1973);

T. Appelquist and J. Carazzone, Phys. Rev. D1 1 (1975) 2856;

Y. Kazama and Y.-P. Yao, Phys. Rev. D25 (1982) 1605;

Y. Kazama, D. G. Unger and Y.-P. Yao, UM-HE-80-36;

D. G. Unger and Y.-P. Yao, Phys. Rev. D25 (1982) 2721;

S. Weinberg, Phys. Lett. $91 \mathrm{~B}$ (1980) 51;

B. Ovrut and H. J. Schnitzer, Phys. Rev. D21 (1980) 3369; D22 (1980) 2518; Nucl. Phys. B179 (1981) 381 ;

T. Hagiwara and N. Nakazawa, Harvard U. HUTP-80/A012;

P. Binetruy and T. Schucker, Nucl. Phys. Bl78 (1981) 293; B178 (1981) 307;

N.-P. Chang et al., Phys. Rev. D25 (1982) 1630; D22 (1980) 1414

[14] S. Weinberg, Phys. Rev. Lett. 29 (1972) 1698; Phys. Rev. D7 (1973) 2888

[15] S. Weinberg, Phys. Lett. 82B (1979) 387

[16] E. Witten, Constraints on supersymmetry breaking, Princeton University preprint

[17] S. Weinberg, talk at Third Workshop on Grand Unification, Chapel Hill, April 1982

[18] P. Ginsparg, Phys. Lett. 112B (1982) 45;

S.-Y. Pi, Harvard U. HUTP-81/A005, Nov. 1981;

K.R. Klinkhamer, Phys. Lett. 110B (1982) 203

[19] K. Sato, Mon. Not. R. Astr. Soc. 195 (1981) 467;

M.B. Einhorn and K. Sato, Nucl. Phys. B180[FS2] (1981) 385;

A.H. Guth and S.-H. Tye, Phys. Rev. Lett. 44 (1980) 631;

A.H. Guth, Phys. Rev. D23 (1981) 347;

A.H. Guth and E.J. Weinberg, Phys. Rev. D23 (1981) 876; M.I.T. CTP \#950, Feb. 1982

[20] A.D. Linde, Phys. Lett. 108B (1982) 389; Lebedev Inst. 82-88, March 1982;

A. Albrecht and P.J. Steinhardt, Phys. Rev. Lett. 48 (1982) 1220;

A. Albrecht, P.J. Steinhardt, M.S. Turner and F. Wilczek, Phys. Rev. Lett. 48 (1982) 1437

[21] L. Hall and I. Hinchliffe, LBL preprint 14806

[22] R. Barbieri et al., Phys. Lett. $115 B$ (1982) 212

[23] P.H. Frampton, H. Georgi and J.E. Kim, Harvard preprint HUTP-82/A025 